Anatomisches Institut (Prof. Dr. A. FALLER) der Universität Fribourg, Schweiz und Anatomisches Institut, Lehrstuhl I (Prof. Dr. A. Oкsche),

der Universität Giessen, Deutschland

\title{
Kombinierte licht- und elektronenmikroskopische Analyse des Zellbildes in den Langerhansschen Inseln des Karpfens*
}

\author{
Adolf FALLER und Rainer LANGE
}

Eingegangen am 18. September, 1969

\begin{abstract}
Wegen der Schwierigkeit einer rein lichtmikroskopischen Differenzierung der Zelltypen in den Langerhansschen Inseln wird in der vorliegenden Arbeit der Versuch gemacht, durch Verwendung schwacher elektronenmikroskopischer Vergrößerungen die Granulamorphologie als zusätzliches Kriterium zu gewinnen. Gerade in der elektronenmikroskopischen Literatur des Karpfenpankreas gibt es Widersprüche (Titlbach, 1966; Watanabe, 1960), die eine solche kombinierte licht- und elektronenmikroskopische Studie verlangen. Die Methodik ist im wesentlichen die früher geschilderte (LANGE, 1967).
\end{abstract}

\section{Material und Methodik}

Inselhaltiges Gewebe wurde aus der Umgebung des Ductus choledochus bei 6 Karpfen nach Dekapitation entnommen und in folgenden Flüssigkeiten fixiert: Bouinsches Gemisch (RomeIs, 1948); Formaldehyd (4\%, neutralisiert, mit Zusatz von Saccharose); Glutaraldehyd (1,5\%, 0,05 M Phosphatpuffer); Acrolein (5\%, 0,1 M Phosphatpuffer); Osmiumtetroxyd (1\%, nach PALADE). Nach Aldehydfixierung wurden die Blöckchen $z$.T. für 2 Std. in $1 \%$ Osmiumtetroxyd nachbehandelt. Die aufgeführten Fixierungen werden im Folgenden durch ihre Anfangsbuchstaben abgekürzt: B, F, F-O, G, G-O, A, A-O, O. Einbettung erfolgte in Paraffin (P), Methakrylat (M), Durkupan ACM (D) oder Vestopal W(V).

Zur kombinierten licht- und elektronenmikroskopischen Auswertung gelangten G-O-M- und G-D-Blöcke, indem Dickschnitte und kurze Dünnschnittbänder im Wechsel angefertigt und im licht- bzw. elektronenmikroskopischen Bild miteinander verglichen wurden. Zur lichtmikroskopischen Anfärbung wurden hierbei Aldehydfuchsin (GABE, 1953) nach Voroxydierung, die $p$-Dimethylaminobenzaldehyd-NitritMethode nach Adams (PEARse, 1960) und die Versilberung nach Winckler (1959) verwendet.

Die Schnitte wurden auf dem Sorvall-MT 1 angefertigt und auf formvarbefilmten Netzen nach $\mathrm{KMnO}_{4}$-Kontrastierung mit dem Hitachi-HS 6 bei 2-3,000 facher Primärvergrößerung mikroskopiert. Methakrylatschnitte wurden vor der lichtmikroskopischen Färbung mit Xylol und Aceton behandelt und vor der elektronenmikroskopischen Untersuchung mit Kohle bedampft.

Weitere lichtmikroskopische Beobachtungen erfolgten mit Versilberung nach

\footnotetext{
* Mit dankenswerter Unterstützung durch den Schweizerischen Nationalfonds (A. F., R. L.) und durch
} die Deutsche Forschungsgemeinschaft (R. L.). 


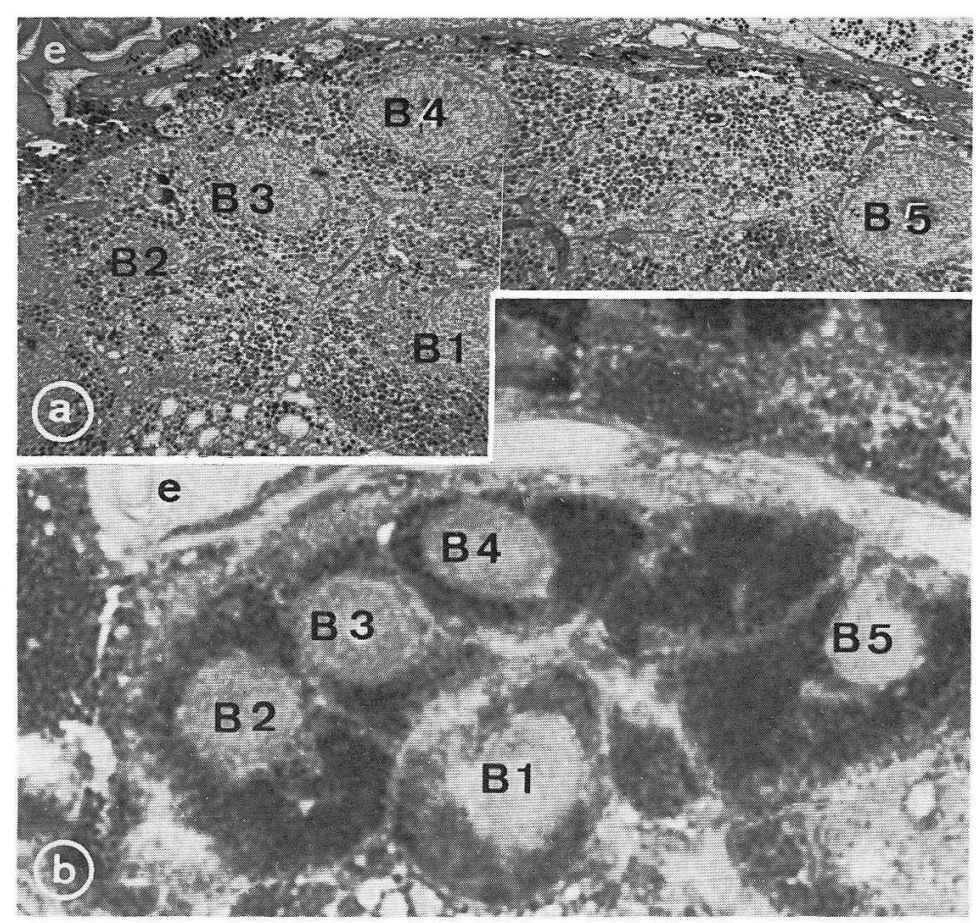

Abb. 1. Gruppe von B-Zellen im elektronenmikroskopischen (a) und lichtmikroskopischen Bild (b). Methodik G-O-M. a: $\mathrm{KMnO}_{1}$-Kontrastierung. b: Aldehydfuchsin nach Oxydation. Vergrößerung etwa $\times 2,000$. Identische Erythrozyten auf beiden Aufnahmen sind mit $e$, identische B-Zellen mit B1-B5 gekennzeichnet.

Lee (1967), Hellman und Hellerström (1960), sowie Methylenblaufärbung (pH 4, $\mathrm{pH}$ 6) mit und ohne vorhergehende Methylierung und Verseifung.

\section{Befunde}

\section{Lichtmikroskopie}

Von den genannten Silbermethoden erwies sich an unserem Material nur diejenige von WINCKLER als geeignet. Metachromasie nach Veresterung und Verseifung ließ sich nur an B-P-Matcrial nachwciscn und somit nicht mit den elektronenmikroskopischen Befunden in Beziehung setzen. Die Zymogengranula der exokrinen Pankreaszellen verhielten sich färberisch erwartungsgemäß wie das B-Zellzytoplasma (Aldehydfuchsin +, Silber -) mit den Ausnahmen, daß die Zymogengranula positiv bei der Reaktion auf Tryptophan (Abb. 2 a) und wesentlich kräftiger bei der Anfärbung mit Methylenblau (pH 4, pH 6) reagierten.

\section{Kombinierte licht-und elektronenmikroskopische Untersuchung}

Auf diesem Weg ließen sich im Inselgewebe 5 verschiedene Zelltypen unterscheiden. Die färberischen und elektronenmikroskopischen Eigenschaften der einzelnen Zelltypen sind in der Tabelle zusammengestellt; bei den lichtmikroskopischen Befunden sind nur kräftige Anfärbung und deutlich negativer Ausfall verzeichnet. 

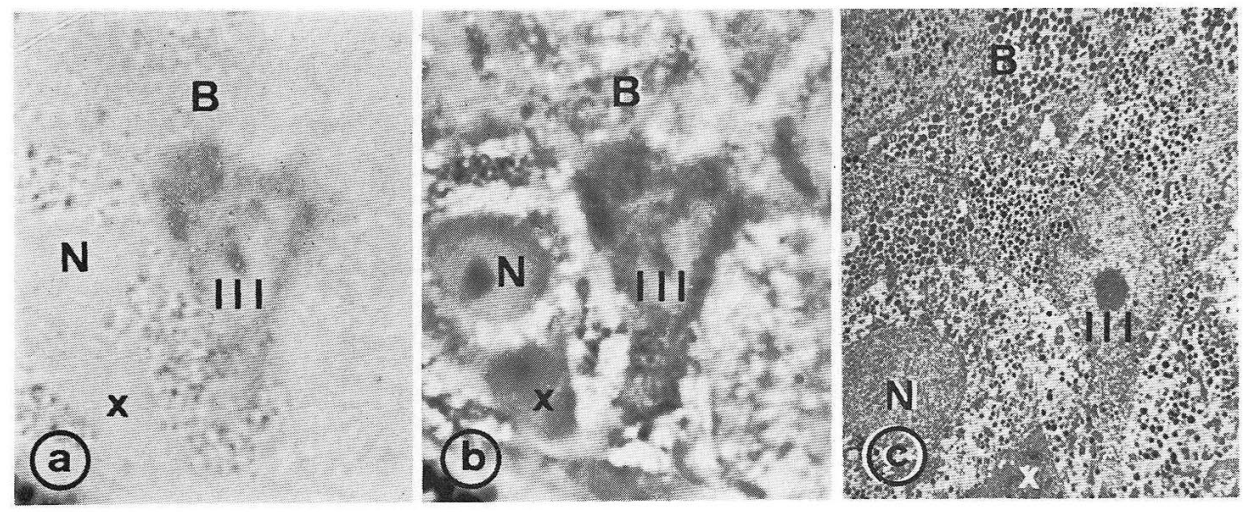

Abb. 2. Inselzelle vom Typ III (tryptophanpositiv): im Hellfeld (a), im Phasenkontrast (b) und im elektronenmikroskopischen Bild (c). Methodik G-D. a und b: p-Dimethylaminobenzaldehyd-NitritMethode auf Tryptophan nach ADAMs. c: $\mathrm{KMnO}_{4}$-Kontrastierung. Vergrößerung etwa $\times 2,000$. Eine Typ-III-Zelle ist auf allen Bildern mit $I I I$, eine andere vakuolisierte 'lyp-III-Zelle mit $N$, eine B-Zelle mit $B$ und eine nicht identifizierte Zelle mit $X$ gekennzeichnet. Auf den lichtmikroskopischen Aufnahmen sind in der linken unteren Ecke Zymogengranula sichtbar.

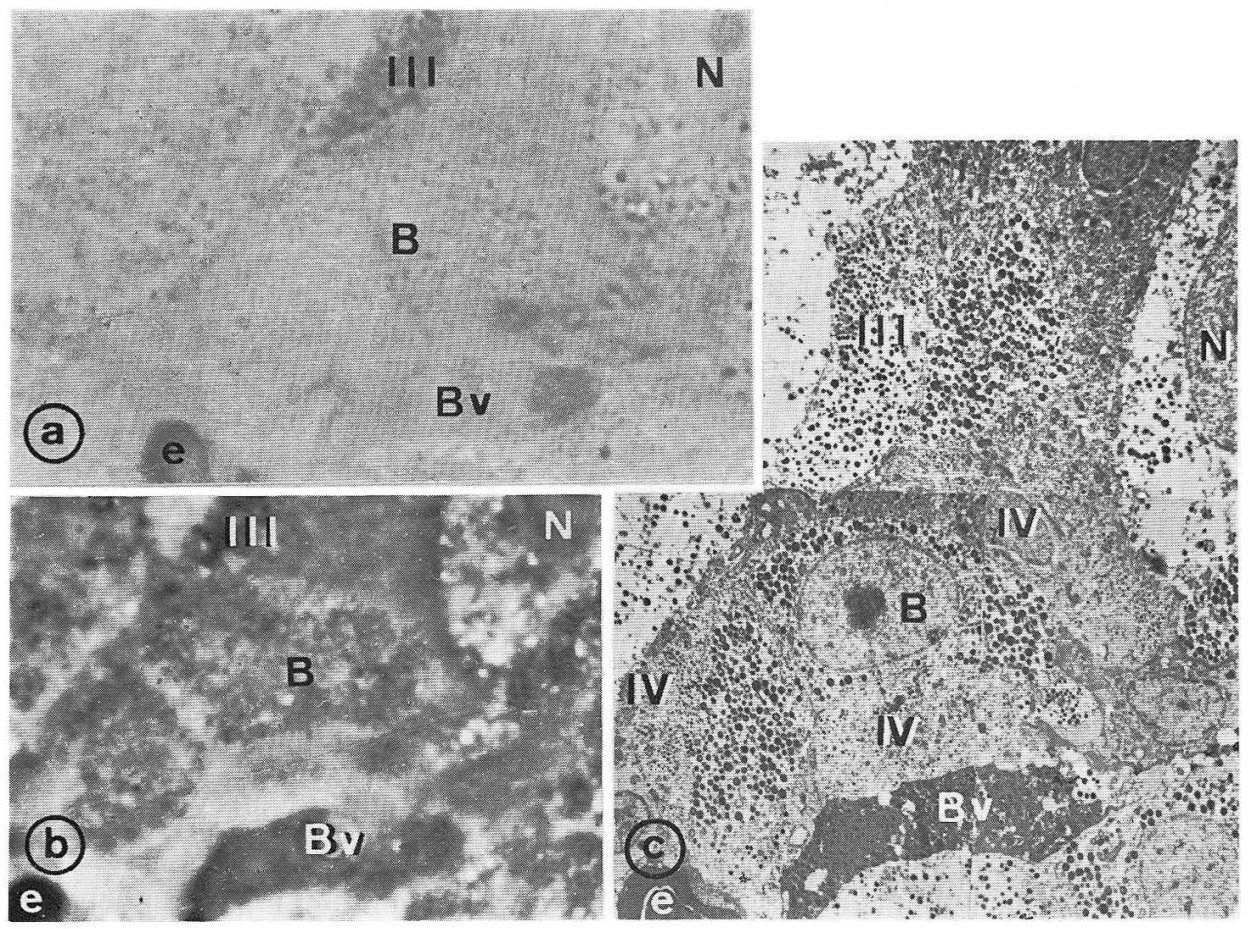

Abb. 3. Gruppe von Inselzellen in dreifacher Darstellung, in Technik, Maßstab und einem Teil der Bezeichnungen wie Abb. 2. Bv kennzeichnet eine B-Zelle mit stark verdichtetem Zytoplasma; diese Zelle tritt auch im Phasenkontrast (b) sehr stark hervor, gibt aber bezeichnenderweise im Hellfeld (a) keine Tryptophanreaktion. e Erythrozyten. IV Zellen vom Typ IV. 


\begin{tabular}{|c|c|c|c|}
\hline \multirow{3}{*}{$\frac{\begin{array}{r}\text { Bezeichnung } \\
\text { des Zelltyps }\end{array}}{\text { B }}$} & \multicolumn{2}{|l|}{ Tabelle } & \multirow[b]{2}{*}{$\begin{array}{l}\text { ad Färbeergebnis } \\
\text { G-O-M }\end{array}$} \\
\hline & $\begin{array}{l}\text { Elektronenmikroskopische Morphologie } \\
\text { nach } \mathrm{KMnO}_{4} \text {-Kontrastierung }\end{array}$ & $\underset{G-D}{\text { Bemerkungen un }}$ & \\
\hline & $\begin{array}{l}\text { Polymorphe Granula mittlerer Elektronen- } \\
\text { dichte, Abb. } 1-3,5 \mathrm{a} \text {. }\end{array}$ & $\begin{array}{l}\text { Adams - } \\
(\mathrm{Abb} .2,3)\end{array}$ & $\begin{array}{l}\text { Gomori }+ \\
(\mathrm{Abb} .1,4)\end{array}$ \\
\hline II & $\begin{array}{l}\text { Wenig elektronendichte Granula, rund; } \\
\text { erweiterter perinukleärer Raum, in Ansamm- } \\
\text { lungen in der Inselperipherie mit wenigen } \\
\text { Granula oder granulareich in den Inseln } \\
\text { verstreut. }\end{array}$ & $\begin{array}{l}\text { nicht in genügen- } \\
\text { der Anzahl identi- } \\
\text { fiziert }\end{array}$ & $\begin{array}{l}\text { bei allen Färbun- } \\
\text { gen praktisch } \\
\text { negativ (Abb. } 4)\end{array}$ \\
\hline III & $\begin{array}{l}\text { Rundliche und längliche Granula der größten } \\
\text { relativen Elektronendichte; Zellen teilweise } \\
\text { stark vakuolisiert (Abb. 2,3). }\end{array}$ & Adams + & \\
\hline IV & Sehr kleine, dicht gelagerte Granula (Abb. 3) & Adams - & \\
\hline $\mathrm{V}$ & $\begin{array}{l}\text { Große Granula mit zentraler Vakuolisierung; } \\
\text { an der Inselperipherie gelegen. }\end{array}$ & nicht identifiziert & $\begin{array}{l}\text { Gomori }+ \\
\text { Silber }+\end{array}$ \\
\hline
\end{tabular}

\section{Elektronenmikroskopische Befunde bei anderen Fixierungen}

Nach O- und A-O-Fixierung wurden granulolytische Zellbilder (Abb. 5 b, c) beobachtet, nicht jedoch nach G- und G-O-Fixierung. Diese granulolytischen Zellbilder sind zweifelsfrei als Nicht-B-Zellen anzusprechen, jedoch ist ihre Klassifizierung als Typ II oder Typ III nicht mit Sicherheit möglich.

\section{Besprechung der Befunde}

Sowohl in licht- als auch in elektronenmikroskopischer Hinsicht bietet die BZelle die geringsten Schwierigkeiten, da sie sich in beiden Fällen gut charakterisieren lässt (Abb. 1, 5 a). Die B-Zellen können im G-O-M- und G-D-Material elektronenmikroskopisch an der Vielgestaltigkeit ihrer Granula zweifelsfrei identifiziert werden. Wir müssen TiтLBAch (1966) in seiner Meinung beipflichten, daß WATANABE (1960) im elektronenmikroskopischen Teil seiner Arbeit über die Langerhansschen Inseln des Karpfens A- und B-Zellen verwechselt hat. Schmale Zelleiber mit stark verdichtetem Zytoplasma, in das typisch geformte B-Granula eingelagert sin d $\mathrm{Abb}$. 3 c: Bv), sind in der Regel völlig negativ bei der Adamsschen Reaktion auf Tryptophan. Dieser Befund bestätigt unsere Beobachtungen am Froschpankreas (LANGE, 1967), wonach der positive Ausfall dieser Reaktion unter den gewählten Bedingungen spezifisch für einen einzigen elektronenmikroskopisch charakterisierten Zelltyp ist (Typ-III-Zelle).

Die Typ-II-Zelle unserer Nomenklatur imponiert auf dem lichtmikroskopisch gefärbten Dickschnitt als chromophobes Element (Abb. 4) und entspricht in dieser Hinsicht möglicherweise dem agranulären Element von FALKMER (1961). Elektronenmikroskopisch zeichnet sich die Typ-II-Zelle jedoch durch eine charakteristische Granulation aus, die in den G-O-M-Schnitten außerordentlich wenig elektronendicht ist. Bemerkenswert ist ferner die Ansammlung der Typ-II-Zellen in Verbänden am Inselrand. Außerdem kamen an den Typ II erinnernde Elemente versteut in den Inseln vor und waren dann meist granulareicher. Leider gelang es uns nicht, TypII-Zellen in unserem G-D-Material sicher zu identifizieren; Typ-II-Zell-Ansammlungen konnten wir in den Inseln dieses Materials nicht finden. Ein Zelltyp, der sich 
elektronenmikroskopisch durch leere Säckchen an Stelle von Granula auszeichnet (TiтlBACH, 1966: D-Zelle), ist in unserem G-O-M- und G-D-Material nicht aufgetreten. Der Verdacht, daß ein Fixierungseinfluß für solche granulolytischen Bilder verantwortlich ist, wird durch unsere Befunde nach Acrolein- und Osmiumfixierung beim
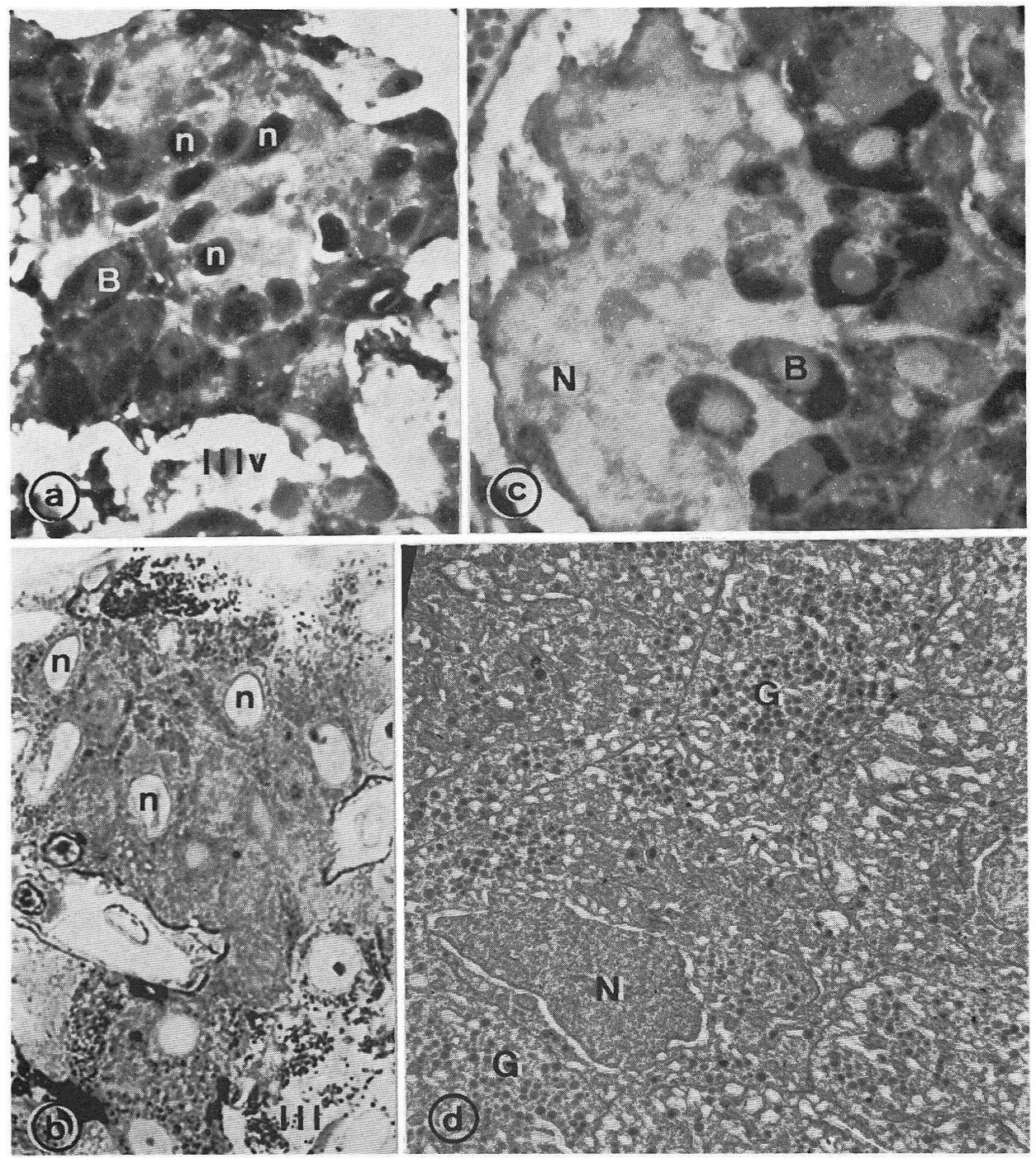

Abb. 4. Typ-II-Zellen nach verschiedenen Färbemethoden. Methodik G-O-M a: Methylenblau; die Typ-II-Zellen heben sich dadurch hervor, daß ihre Kerne $(n)$ sich stärker als ihre Zelleiber anfärben. B B-Zelle; IIIv vakuolisierte Zelle vom Typ III. Vergrößerung $\times 1,000$. b: Versilberung nach WinckLeR. Im Originalpräparat sind in diesem Gesichtsfeld nur Gelb- und Brauntöne vorhanden, keine Schwärzung. In den Typ-II-Zellen $(n)$ zarte Zytoplasmagranulation. III TypIII-Zelle. $\times 1,200$. c: Aldehydfuchsin nach Oxydation. Links Gruppe von Typ-II-Zellen mit praktisch ungefärbten Zelleibern, rechts B-Zellen $(B)$ und Typ-III-Zellen. $N$ kennzeichnet den Zellkern derselben Zelle wie auf der elektronenmikroskopischen Abbildung $(d) . \times 1,000$ d: Elektronenmikroskopische Aufnahme nach $\mathrm{KMnO}_{4}$-Kontrastierung. Beachte die zarten Granula $(G)$. Vergrößerung etwa. $\times 5,000$ 


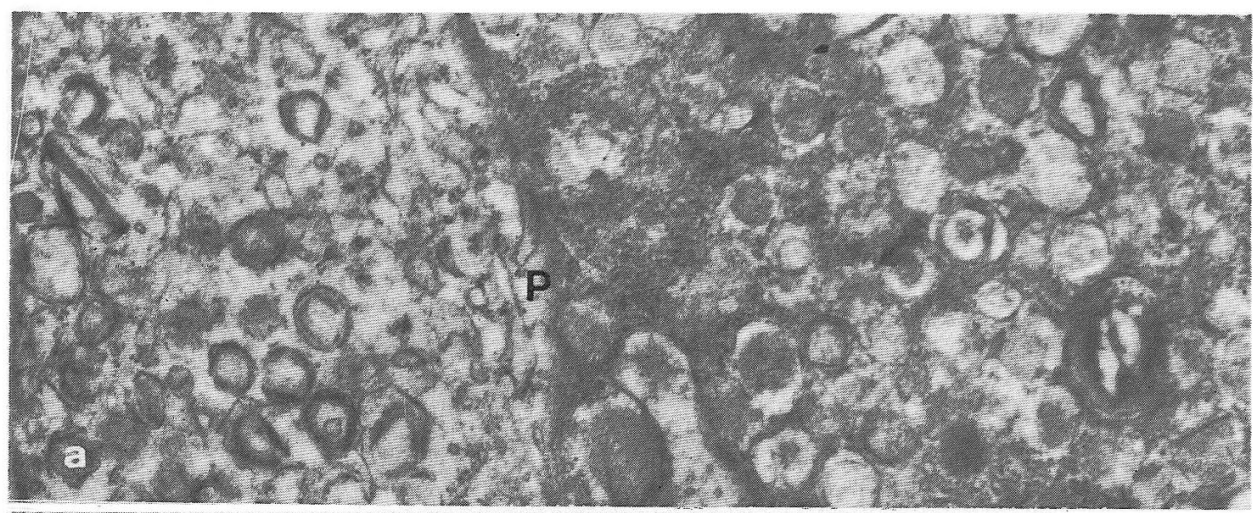

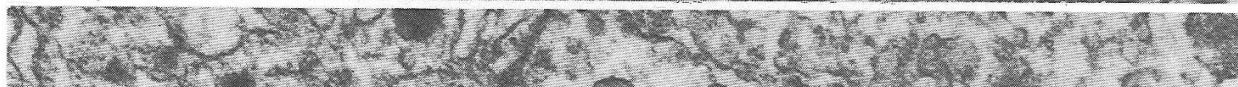

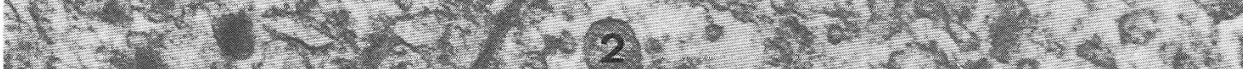

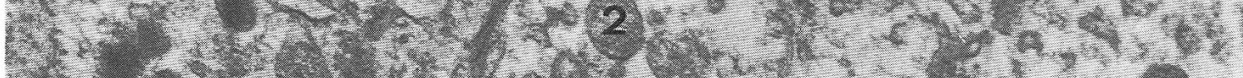

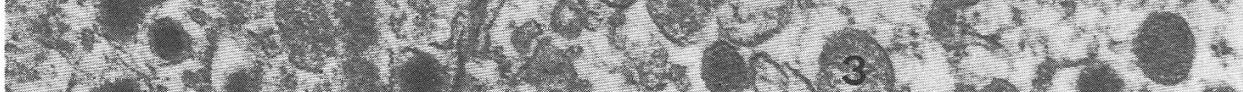

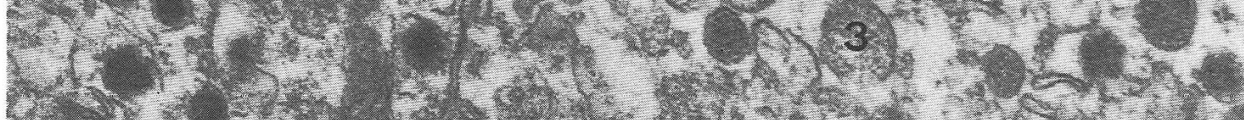
f. (3)

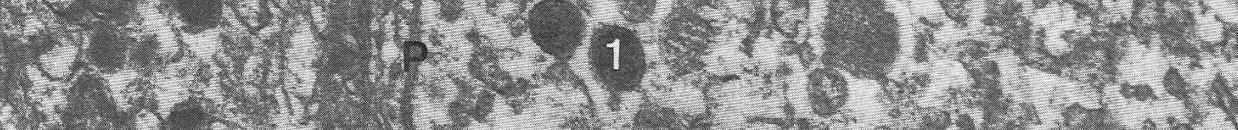

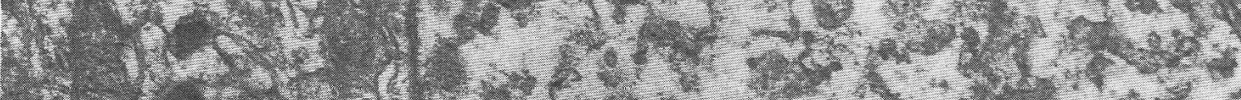

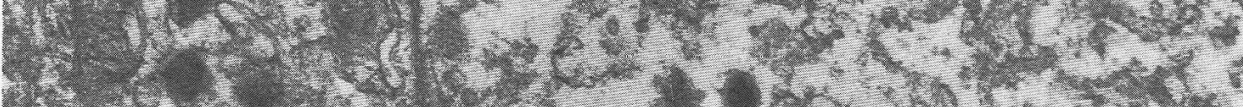

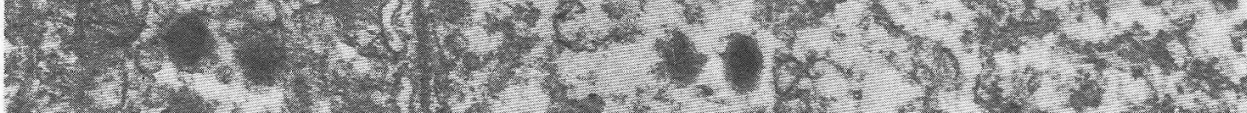

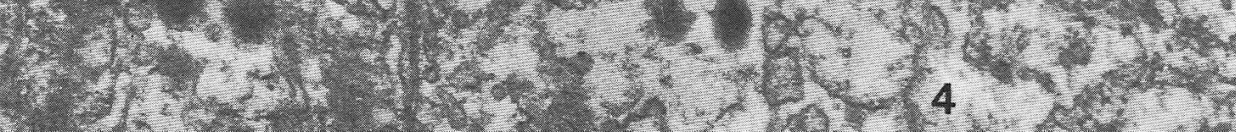

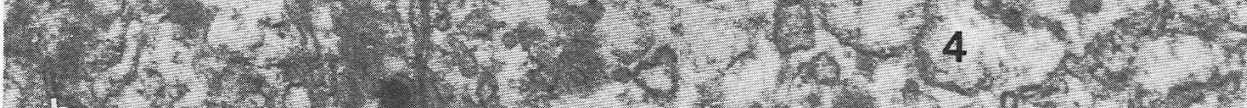

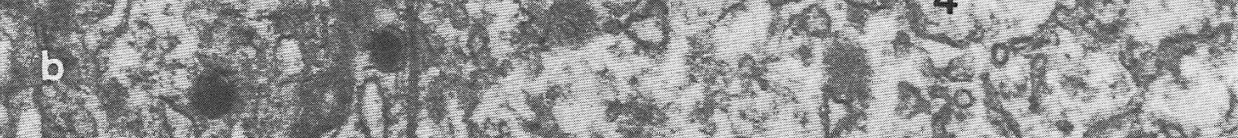

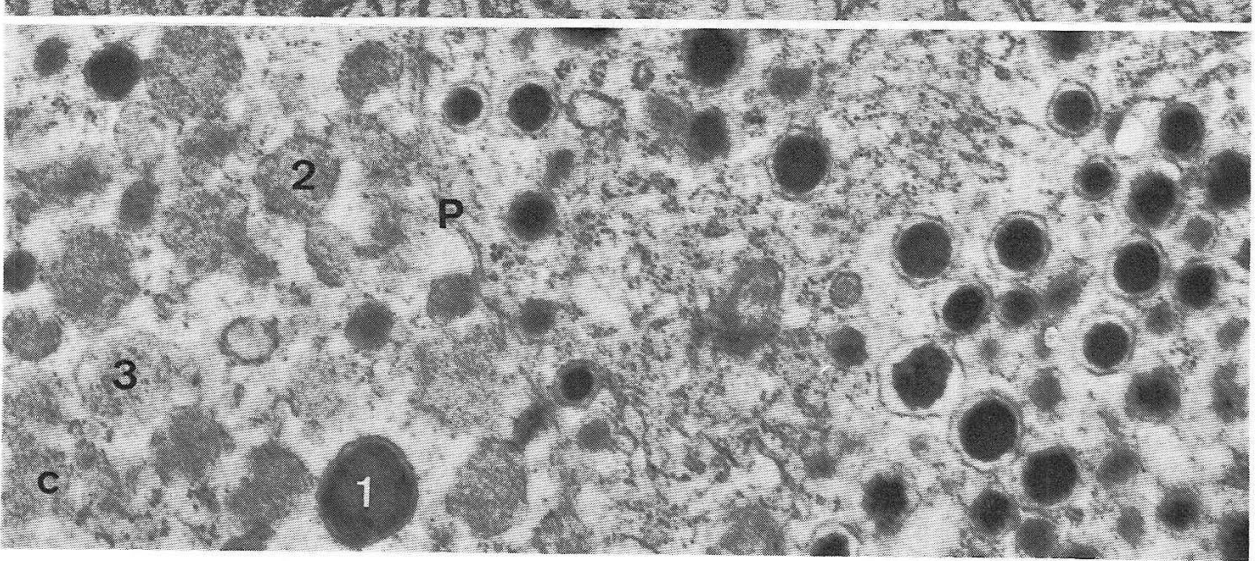

Abb. 5. 
Karpfen (Abb. 5 b, c) und beim Frosch (LANGe, 1968) bekräftigt. Auch Titlbach (1966) bildet D-Zellen nur nach Osmiumfixierung ab.

Als Typ-III-Zelle wird in Anlehnung an die Befund beim Frosch (Lange, 1967) das Element bezeichnet, das eine positive Reaktion beim Tryptophannachweis nach ADAms gibt. Nur wenn es gelingt, die Ergebnisse der G-D-Technik und der G-O-MTechnik in Beziehung zu setzen, werden sich die Typ-III-Zell-Befunde in die Tabelle einordnen lassen. Sowohl im G-D- als auch im G-O-M-Material kommt ein Zelltyp mit rundlichen, teilweise länglich ausgezogenen Granula vor, die kleiner und deutlich elektronendichter als B-Granula sind. Die Vertreter dieses Zelltyps können ein normal dichtes Zytoplasma aufweisen. Ihr Zelleib kann aber auch die Einlagerung großer, leer erscheinender Vakuolen stark aufgelockert sein. Schließlich können diese Zellen $\mathrm{zu}$ schmalen, außerordentlich verdichteten Elementen umgestaltet erscheinen: Alle 3 Formen geben - soweit bei den vakuolig aufgetriebenen Zellen mit verstreut liegenden Granula sicher beurteilbar (Abb. 2 a) - im G-D-Material positive Tryptophanreaktion. An G-O-M-Material wurde die Methode von ADAms wegen früherer negativer Ergebnisse (LANGE, 1967) nicht durchgeführt. Wesentlich ist nun die Frage, ob das Kriterium der relativen Elektronendichte der Granula bei $\mathrm{KMnO}_{4}$-Kontrastierung nach verschiedenen Fixierungen und Einbettungen (G-D und G-O-M) als Charakteristikum für die Abgrenzung eines Zelltypus genügt. Mangels anderer Anhaltspunkte möchten wir diese Frage bejahen. Die vakuolig aufgetriebenen Formen der Typ-III-Zellen imponieren lichtmikroskopisch als granulaarmes bzw. chromophobes Element. Interessant erscheint uns, daß beim Frosch im Gegensatz zu den für den Karpfen beschriebenen Verhältnissen im G-O-Material der Typ III (tryptophanhaltig) die am wenigsten elektronendichten Granula besitzt.

Die Typ-IV-Zellen, oft nur in Form von Anschnitten zarter Zellausläufer zu beobachten, kommen auch als kernhaltige Schnittbilder vor. Sie liegen gelegentlich in kleinen Gruppen zusammen (Abb. 3) und zeichnen sich durch ihre außerordentlich kleinen Granula aus. Anschnitte von kleinen Ausläufern solcher Zellen gleichen granulahaltigen marklosen Nervenfasern.

Die Typ-V-Zellen, ebenso rätselhaft wie der vorhergehende Zelltyp, kommen anscheinend nur an der Inselperipherie vor. Ihre Granula erinnern an Zymogengranula, mit denen sie Größe und Elektronendichte des Materials gemeinsam haben, unterscheiden sich von diesen jedoch dadurch, daß sie typischerweise eine leer erscheinende Vakuole enthalten. Einzig bei den Typ-V-Zellen wurde mit der Silbermethode von WiNcKLER eine Schwärzung der Granula (im Gegensatz zu weitverbreiteter Bräunung) erzielt. Ob es sich bei den Typ-V-Zellen um eigentliche Inselelemente oder um veränderte exokrine Zellen handelt, kann nicht entschieden werden.

\section{Zusammenfassung}

In einer Untersuchung der Langerhansschen Inseln des Karpfens, die sich hauptsächlich auf die gleichzeitige licht- und elektronenmikroskopische Betrachtung

Abb. 5. Elektronenmikroskopische Detailaufnahmen. a: Methodik O-V, Uranylacetat. B-Zellen, links mit hellem, rechts mit verdichtetem Zytoplasma. $\times 30,000$. b: Methodik O-V, Phosphorwolframsäurekontrastierung. In der Zelle rechts schlechte Granulakonservierung nach Osmiumfixierung: die Stadien zunehmender Granulolyse sind mit 1-4 bezeichnet. $\times 30,000$ c: Methodik $\mathrm{A}-\mathrm{O}-\mathrm{V}, \mathrm{Pb}-$ Kontrastierung. In der Zelle links schlechte Granulakonservierung (vgl. Abb. 5 b), hier nach Acroleinfixierung. $P$ Plasmalemm. $\times 30,000$. 
derselben Zellen auf benachbarten Dick- und Dünnschnitten stützt, werden folgende Zelltypen abgegrenzt:

1. B-Zelle mit sehr vielgestaltigen Granula und typischer lichtmikroskopischer Anfärbung (Gomori +, negativ bei Versilberung und Tryptophannachweis).

2. Typ-II-Zelle mit sehr wenig elektronendichten Granula, negativ bei allen lichtmiktroskopischen Färbungen, die versucht wurden. Anhand dieser Zellen wird das Problem der agranulären Zellen erwähnt und auf Fixierungsartefakte (Osmium-, Acroleinfixierung) hingewiesen.

3. Typ-III-Zelle mit rundlichen Granula der größten relativen Elektronendichte und positiver Reaktion beim Tryptophannachweis nach ADAms.

4. Typ-IV-Zelle mit sehr kleinen Granula; Anschnitte solcher Zellen gleichen gelegentlich marklosen Nervenfasern.

5. Typ-V-Zellen, die mit ihren Granula den exokrinen Pankreaszellen ähneln und durch die Silbermethode nach WINCKLER geschwärzt werden.

\section{Literatur}

Falkmer, S.: Experimental diabetes reseach in fish. On the morphology and physiology of the endocrine pancreatic tissue of the marine teleost Cottus scorpius with special reference to the role of glutathione in the mechanism of alloxan diabetes using a modified nitroprusside method. Acta endocrinol. Suppl. 59 (1961).

Gabe, M.: Sur quelques applications de la coloration par la fuchsine-paraldéhyde. Bull. Microsc. appl. 3: 153-162 (1953).

Hellman, B. and C. Hellerström: The islets of Langerhans in ducks and chickens with special reference to the argyrophilic reaction. Z. Zellforsch. 52: 278-290 (1960).

Lange, R.: Licht- und elektronenmikroskopische Identifizierung der Zelltypen im Inselapparat des Frosches Rana ridibunda. Z. Zellforsch. 82: 156-172 (1967).

-: Über fixationsbedingte Unterschiede in der elektronenmikroskopischen Morphologie der Zelltypen im Inselapparat des Frosches Rana ridibunda. Z. Zellforsch. 86: 238-251 (1968).

Lee, D.: Identification of argyrophilic cells in pancreatic islets by light and electron microscopy in osmium-fixed plastic-embedded sections. Z. Zellforsch. 77: 1-7 (1967).

Pearse, A. G. E.: Histochemistry. Theoretical and applied. 2nd ed. London, Churchill Ltd., 1960.

Romeis, B.: Mikroskopische Technik. 15. Aufl. München, R. Oldenbourg, 1948.

Titlbach, M.: Feinstruktur der Langerhansschen Inseln bei Cyprinus carpio L. Z. mikr.-anat. Forsch. 75: 184-197 (1966).

Watanabe, A.: Histologische, cytologische und elektronenmikroskopische Untersuchungen über Langerhansschen Inseln der Knochenfische, insbesondere des Karpfens. Arch. histol. jap. 19: 1-52 (1960).

Winkler, G.: A propos de la technique de l'imprégnation argentique de Marsland, Glees et Erikson. Arch. Anat. Histol. Embryol. norm. pathol. 42: 231-241 (1959). 